\title{
Bifurcation of critical periods from Pleshkan's isochrones
}

\author{
M. Grau and J. Villadelprat \\ Departament de Matemàtica, \\ Universitat de Lleida, Lleida, Spain \\ Departament d'Enginyeria Informàtica i Matemàtiques, \\ Universitat Rovira i Virgili, Tarragona, Spain
}

\begin{abstract}
Pleshkan proved in 1969 that, up to a linear transformation and a constant rescaling of time, there are four isochrones in the family of cubic centers with homogeneous nonlinearities $\mathscr{C}_{3}$. In this paper we prove that if we perturb any of these isochrones inside $\mathscr{C}_{3}$, then at most two critical periods bifurcate from its period annulus. Moreover we show that, for each $k=0,1,2$, there are perturbations giving rise to exactly $k$ critical periods. As a byproduct, we obtain a partial result for the analogous problem in the family of quadratic centers $\mathscr{C}_{2}$. Loud proved in 1964 that, up to a linear transformation and a constant rescaling of time, there are four isochrones in $\mathscr{C}_{2}$. We prove that if we perturb three of them inside $\mathscr{C}_{2}$, then at most one critical period bifurcates from its period annulus. In addition, for each $k=0,1$, we show that there are perturbations giving rise to exactly $k$ critical periods. The quadratic isochronous center that we do not consider displays some peculiarities that are discussed at the end of the paper.
\end{abstract}

\section{Introduction and statement of the results}

In this paper we study the period function of the centers of planar vector fields with homogeneous nonlinearities, more concretely, differential systems of the form

$$
\left\{\begin{array}{l}
\dot{x}=-y+P_{n}(x, y) \\
\dot{y}=x+Q_{n}(x, y)
\end{array}\right.
$$

where $P_{n}(x, y)$ and $Q_{n}(x, y)$ are homogeneous real polynomials of degree $n$. We denote by $\mathscr{H}_{n}$ the family of vector fields of the above form and by $\mathscr{C}_{n}$ the subset of those systems in $\mathscr{H}_{n}$ with a center at the origin. Finally, $\mathcal{I}_{n}$ stands for the set of nonlinear isochrones in $\mathscr{H}_{n}$. Accordingly $\mathcal{I}_{n} \subset \mathscr{C}_{n} \subset \mathscr{H}_{n}$. We restrict ourselves to the cases $n=2$ and $n=3$, that are the degrees for which the centers and the isochrones have been classified.

Recall that the period annulus of a center is the biggest punctured neighbourhood foliated by periodic orbits and in what follows we shall denote it by $\mathcal{P}$. Compactifying $\mathbb{R}^{2}$ to the Poincaré disc, the boundary of $\mathcal{P}$ has two connected components: the center itself and a polycycle. We call them respectively the inner and outer boundary of the period annulus. The period function of the center assigns to each periodic orbit $\gamma$ in $\mathcal{P}$ its period. If all the periodic orbits in $\mathcal{P}$ have the same period, then the center is called isochronous. Since the period function is defined on the set of periodic orbits in $\mathcal{P}$, usually the first step is to parameterize

2000 AMS Subject Classification: 34C23; 37C10;37C27.

Key words and phrases: critical period; isochronous center; period function; bifurcation; unfolding.

M. Grau is partially supported by the MCYT/FEDER grant number MTM2005-06098-C02-02. J. Villadelprat is partially supported by the CONACIT through the grant 2005-SGR-00550 and by the DGES through the grant MTM-2005-06098-C02-1. 
this set, let us say $\left\{\gamma_{s}\right\}_{s \in(0,1)}$, and then one can study the qualitative properties of the period function by means of the map $s \longmapsto$ period of $\gamma_{s}$, which is analytic on $(0,1)$. The critical periods are the critical points of this function and their number, character (maximum or minimum) and distribution do not depend on the particular parameterization of the set of periodic orbits used. We are interested in the bifurcation of critical periods. Roughly speaking, the disappearance or emergence of critical periods as we perturb a center. There are three different situations to study (see [19] for details):

(a) Bifurcation of the period function from the inner boundary of $\mathcal{P}$ (i.e., the center itself).

(b) Bifurcation of the period function from $\mathcal{P}$.

(c) Bifurcation of the period function from the outer boundary of $\mathcal{P}$ (i.e., the polycycle).

In this paper we are only concerned with bifurcation of the period function from $\mathcal{P}$, i.e. case $(b)$, and when we perturb an isochronous center. To be more precise, we study the bifurcation of critical periods from $\mathcal{P}$ of the isochrones in $\mathcal{I}_{n}$ when perturbed inside $\mathscr{C}_{n}$. Our initial motivation was to study this problem for $n=3$ and the result we have obtained is stated in Theorem $\mathrm{A}$ below. As a byproduct of the tools we develop to prove it, we get a partial result of the problem for $n=2$, see Theorem $\mathrm{B}$.

As the authors explain in [7, 8] many problems dealing with the period function and critical periods are the counterpart of problems about the Poincaré return map and limit cycles. The problem studied here is the counterpart of the question of how may limit cycles bifurcate from a center of an integrable system when perturbed in a non-conservative direction. The return map of the unperturbed system is the identity and one asks for the number of fixed points that persist. In our case the map that we perturb is constant (the period function of an isochronous center) and we ask for the number of zeros of the derivative that persist. Let us comment in this setting that the bifurcation of the period function from $\mathcal{P}$, i.e., case $(b)$, when the unperturbed center is not isochronous is out of reach for the moment. Its counterpart for limit cycles is the so-called blue-sky bifurcation, which is usually undetectable.

The classification of $\mathscr{C}_{3}$ and $\mathcal{I}_{3}$ is due to Malkin [16] and Pleshkan [22, respectively. The latter proved that, up to a linear change of coordinates and a constant rescaling of time (see [5, 21]), there are four isochronous centers in $\mathscr{H}_{3}$ :

$$
\begin{aligned}
& \left(S_{1}^{*}\right) \quad\left\{\begin{array}{l}
\dot{x}=-y-3 x^{2} y+y^{3}, \\
\dot{y}=x+x^{3}-3 x y^{2},
\end{array}\right. \\
& \left(S_{3}^{*}\right)\left\{\begin{array} { l } 
{ \dot { x } = - y + 3 x ^ { 2 } y , } \\
{ \dot { y } = x - 2 x ^ { 3 } + 9 x y ^ { 2 } , }
\end{array} \quad ( \overline { S } _ { 3 } ^ { * } ) \quad \left\{\begin{array}{l}
\dot{x}=-y+x^{2} y, \\
\dot{y}=x+x y^{2},
\end{array}\right.\right. \\
& \hline \dot{x}=-y-3 x^{2} y,
\end{aligned}
$$

The first result that we shall prove is the following. Let us point out that in its statement $\mathcal{P}$ refers to the period annulus of the center at the origin.

Theorem A. If we perturb $\left(S_{1}^{*}\right),\left(S_{2}^{*}\right),\left(S_{3}^{*}\right)$ or $\left(\bar{S}_{3}^{*}\right)$ inside $\mathscr{C}_{3}$, then at most two critical periods bifurcate from $\mathcal{P}$. Moreover, for each $k=0,1,2$, there are perturbations that give rise to exactly $k$ critical periods bifurcating from $\mathcal{P}$.

To be more precise, if $X_{0}$ denotes the vector field associated to one of the systems in (2), then we consider the unfolding given by $X_{\varepsilon}=X_{0}+Y_{\varepsilon}$ with

$$
Y_{\varepsilon}(x, y):=\left(a(\varepsilon) x^{2} y+b(\varepsilon) y^{3}\right) \partial_{x}+\left(c(\varepsilon) x^{3}+d(\varepsilon) x y^{2}\right) \partial_{y}
$$

where $a, b, c$ and $d$ are analytic functions vanishing at $\varepsilon=0$. Since $X_{\varepsilon}$ is reversible, it is clear that it belongs to $\mathscr{C}_{3}$ for all $\varepsilon$. The fact that there is no other perturbation with this property follows from Malkin's result (see also [23]). Of course the problem under consideration only makes sense in case that the perturbation is not isochronous itself. This, let us say, isochronous direction of perturbation that we must avoid is 
$d+3 c=a+3 c=b-c=0$ for $\left(S_{1}^{*}\right) ; b=c=a-d=0$ for $\left(S_{2}^{*}\right) ;$ and $b=2 a+3 c=2 d+9 c=0$ for $\left(S_{3}^{*}\right)$ and $\left(\bar{S}_{3}^{*}\right)$. As we will see, the existence of such a direction prevents the parameters in the perturbation from acting independently and it explains the reason why three critical periods cannot appear even though we consider a 4-parameter unfolding.

There are some previous results related to Theorem $\mathrm{A}$ that should be referred. Rousseau and Toni prove in [23] that if we perturb any isochronous center in (2) inside $\mathscr{C}_{3}$, then at most two critical periods bifurcate from the inner boundary of $\mathcal{P}$. They also study the perturbation of the linear (isochronous) center $X_{0}=-y \partial_{x}+x \partial_{y}$. In this case there are three different unfoldings of $X_{0}$ inside $\mathscr{C}_{3}$ and the authors prove that they give rise, respectively, to at most 0,1 and 3 critical periods bifurcating from the inner boundary of $\mathcal{P}$. In 4 the authors also study unfoldings of the linear center. They provide some lower bounds for the maximum number of critical periods that can bifurcate from $\mathcal{P}$.

The classification of $\mathscr{C}_{2}$ and $\mathcal{I}_{2}$ is due to Dulac [6] and Loud [15, respectively. The latter proved that, up to a linear change of coordinates and a constant rescaling of time, there are four isochronous centers in $\mathscr{H}_{2}$ :

$$
\begin{aligned}
& \left(S_{1}\right) \quad \begin{cases}\dot{x}=-y+x y, \\
\dot{y}=x-\frac{1}{2} x^{2}+\frac{1}{2} y^{2},\end{cases} \\
& \left(S_{3}\right)\left\{\begin{array}{l}
\dot{x}=-y+x y, \\
\dot{y}=x+\frac{1}{4} y^{2},
\end{array}\right.
\end{aligned}
$$

Concerning these systems and the period annulus $\mathcal{P}$ of the isochronous center at the origin, we prove the following result:

Theorem B. If we perturb $\left(S_{2}\right),\left(S_{3}\right)$ or $\left(S_{4}\right)$ inside $\mathscr{C}_{2}$, then at most one critical period bifurcates from $\mathcal{P}$. Moreover, for each $k=0,1$, there are perturbations that give rise to exactly $k$ critical periods bifurcating from $\mathcal{P}$.

The perturbation of the isochrones $\left(S_{2}\right),\left(S_{3}\right)$ and $\left(S_{4}\right)$ inside $\mathscr{C}_{2}$ can be studied with the same unfolding. Indeed, if $X_{0}$ is the associated vector field to one of these three systems, then it suffices to consider $X_{\varepsilon}=$ $X_{0}+Y_{\varepsilon}$ with

$$
Y_{\varepsilon}(x, y):=b(\varepsilon) x y \partial_{x}+\left(d(\varepsilon) x^{2}+f(\varepsilon) y^{2}\right) \partial_{y}
$$

where $b, d$ and $f$ are analytic functions vanishing at $\varepsilon=0$. Clearly $X_{\varepsilon}$ has a center at the origin because the vector field is reversible. The fact that there is not any other perturbation of $X_{0}$ inside $\mathscr{C}_{2}$ follows from Dulac's result. On the contrary, to study the perturbation of $\left(S_{1}\right)$ inside $\mathscr{C}_{2}$ it is necessary to consider two unfoldings. The first one corresponds to the perturbation inside the reversible centers and the second one to the perturbation inside the so-called generalized Lotka-Volterra centers (see [3] for details). However this is not the reason why Theorem $\mathrm{B}$ does not contemplate the perturbation of $\left(S_{1}\right)$. We explain what happens in this case at the end of the paper.

There are previous results related with Theorem B that must be referred. Chicone and Jacobs 3 prove that if we perturb any isochronous center in (4) inside $\mathscr{C}_{2}$, then at most one critical period bifurcates from the inner boundary of $\mathcal{P}$ (i.e., the center). They consider as well the perturbation of the linear isochronous center, for which the upper bound is two. The bifurcation of critical periods from the outer boundary of $\mathcal{P}$ (i.e, the polycycle) is partially studied in [18, 19, 20]. (In [19] the authors present a conjectural bifurcation diagram of the period function of any quadratic center in the Loud form. We reproduce this diagram at the end of the paper, see Figure 1, where we shall comment how Theorem $B$ and this conjecture fit together.) Gasull and $\mathrm{Yu} 10$ prove Theorem B assuming that the perturbation (5) is linear in $\varepsilon$ (or, as they say, perturbations up to first order in $\varepsilon$ ). Let us note moreover that system $\left(S_{4}\right)$ has another isochronous center apart from the one at the origin and that the authors in 10 study the simultaneous bifurcation of critical periods from both period annuli. In this paper we restrict our study to the bifurcation of critical periods from the period annulus of the center at the origin. Finally, the assertion in Theorem $\mathrm{B}$ corresponding to the isochronous center $\left(S_{2}\right)$ is proved in [11. 
In view of Theorems $\mathrm{A}$ and $\mathrm{B}$, and all the previous results we refer above, one may pose the following question for the family $\mathscr{H}_{n}$ of vector fields with homogeneous nonlinearities of degree $n$ : Is it true that at most $n-1$ critical periods can bifurcate from a nonlinear isochronous center when perturbed inside $\mathscr{C}_{n}$ ?

The proof of Theorem $\mathrm{A}$ is given in Section 3 and it is based on a recent result which appears in [12, see the forthcoming Proposition 3.10. This recent result is a criterion for a collection of Abelian integrals to have a Chebyshev property and it provides, in many cases, a shortcut with respect to the classical methods for the same issue. The proof for system $\left(S_{1}^{*}\right)$ follows the classical approach and, thus, it is much longer than the proof for the other three cases. The proof of Theorem B is given in Section 4 . The following section contains the definitions and previous results needed to prove our results.

\section{Definitions and notation}

Let $\left\{X_{\varepsilon}\right\}$ be an analytic family of planar vector fields with a center at the origin for all $\varepsilon \approx 0$ and assume that the center is isochronous for $\varepsilon=0$. Let $\mathcal{P}$ be the period annulus of the center of $X_{0}$ (i.e., the biggest punctured neighbourhood of the origin foliated by periodic orbits) and let $\xi: \mathcal{I} \longrightarrow \mathbb{R}^{2}$ be an analytic transversal section to $X_{0}$ in $\mathcal{P}$, with $\mathcal{I}$ an open real interval. Let $T(s ; \varepsilon)$ be the period of the periodic orbit of $X_{\varepsilon}$ passing through the point $\xi(s) \in \mathcal{P}$ whenever is defined. (Here we use that the curve given by the graphic of $\xi$ is still a transversal section to $X_{\varepsilon}$ for $\varepsilon \approx 0$.) We can choose, for instance, the transversal section to be a solution of the vector field orthogonal to $X_{0}$ and in this case $\mathcal{I}$ is its maximal interval of definition.

Definition 2.1 We say that $k$ critical periods bifurcate from $\mathcal{P}$ in the family $\left\{X_{\varepsilon}\right\}$ as $\varepsilon \rightarrow 0$ if there exist $k$ functions $s_{i}(\varepsilon)$, continuous in a neighbourhood of $\varepsilon=0$, and such that $T^{\prime}\left(s_{i}(\varepsilon) ; \varepsilon\right) \equiv 0$ and $s_{i}(0)=s_{i}^{\star} \in \mathcal{I}$ for each $i=1,2, \ldots, k$.

Since $T(s ; \varepsilon)$ is analytic for $\varepsilon \approx 0$, we can consider its Taylor development at $\varepsilon=0$, say

$$
T(s ; \varepsilon)=\sum_{i=0}^{\infty} T_{i}(s) \varepsilon^{i} .
$$

Note that in fact $T_{0}$ is constant because by assumption the center is isochronous for $\varepsilon=0$. Then, if the center of $X_{\varepsilon}$ is non-isochronous for $\varepsilon \neq 0$, there exists some $\ell \geqslant 1$ such that

$$
T^{\prime}(s ; \varepsilon)=T_{\ell}^{\prime}(s) \varepsilon^{\ell}+o\left(\varepsilon^{\ell}\right)
$$

where $T_{\ell}^{\prime}$ is not identically zero and the remainder is uniform in $s$ on each compact subinterval of $\mathcal{I}$. In this case, from Definition 2.1 it follows that $T_{\ell}^{\prime}\left(s_{i}^{\star}\right)$ for $i=1,2, \ldots, k$. Consequently, by applying the Weierstrass Preparation Theorem, the number of zeros of $T_{\ell}^{\prime}(s)$ for $s \in \mathcal{I}$, counted with multiplicities, provides an upper bound for $k$. A lower bound for $k$ is given by the number of simple zeros of $T_{\ell}^{\prime}(s)$ in $\mathcal{I}$ by using the Implicit Function Theorem.

Remark 2.2 The number of critical periods that bifurcate from $\mathcal{P}$ as $\varepsilon \rightarrow 0$ does not depend on the particular parameterization of the set of periodic orbits in $\mathcal{P}$ used. If two of these parameterizations yield to $T(s ; \varepsilon)$ and $\widehat{T}(s ; \varepsilon)$, then there exists a diffeomorphism $\zeta: \widehat{\mathcal{I}} \longrightarrow \mathcal{I}$ such that $T(\zeta(s) ; \varepsilon)=\widehat{T}(s ; \varepsilon)$. Following the obvious notation, this implies that $\widehat{T}_{\ell}^{\prime}(s)=\zeta^{\prime}(s) T_{\ell}^{\prime}(\zeta(s))$.

Definition 2.3 On account of the above observation, from now on we shall work with a transversal section constructed in the following way. We take a vector field $U_{0}$ analytic on $\mathcal{P} \cup\{(0,0)\}$, transversal to $X_{0}$ at $\mathcal{P}$ and such that $\left[X_{0}, U_{0}\right] \equiv 0$. In the literature such a vector field is called a commutator of $X_{0}$ and it always exists in a neighbourhood of an isochronous center, see 24. Here we require more; it must be analytic on $\mathcal{P} \cup\{(0,0)\}$, but in all the examples that we shall study such a commutator exists. Then we define $T(s, \varepsilon)$ 
exactly as before taking a solution of $U_{0}$ as the transversal section $\xi$. More concretely, we choose any $q \in \mathcal{P}$ and we consider the solution $\psi(s ; q)$ of $U_{0}$ with $\psi(0 ; q)=q$. Then we define $T(s ; \varepsilon)$ as the period of the periodic orbit of $X_{\varepsilon}$ passing through $\xi(s):=\psi(s ; q)$.

Given an analytic family of functions $\left\{g_{\varepsilon}\right\}$ we write its Taylor series at $\varepsilon=0$ as $g(x, y)=\sum_{i=0}^{\infty} g_{i}(x, y) \varepsilon^{i}$. We shall also use the notation $j^{k}(g)=\sum_{i=0}^{k} g_{i} \varepsilon^{i}$ for its $k$-jet with respect to $\varepsilon$. (This notation extends for vector fields in the obvious way.) Moreover, in what follows, we shall say that two vector fields $X$ and $Y$ are conjugated if there exists a change of coordinates $\Phi$ transforming $X$ to $Y$, i.e., $\Phi^{*} X=Y$, where

$$
\left(\Phi^{*} X\right)(p)=(D \Phi)_{p}^{-1}(X \circ \Phi(p)) .
$$

Then we say that $\Phi$ conjugates $X$ and $Y$. A classical result shows that $X$ has an isochronous center at $p_{0}$ if and only if there exists an analytic diffeomorphism $\Phi$ on a neighbourhood of $p_{0}$ that conjugates $X$ with its linear part at $p_{0}$. In this case $\Phi$ is called a linearization.

\section{Proof of Theorem $\mathrm{A}$}

Let $X_{0}$ denote the vector field associated to one of the systems listed in (2) and $\mathcal{P}$ be the period annulus of the isochronous center at the origin. Let $U_{0}$ be a commutator of $X_{0}$ which is analytic on $\mathcal{P} \cup\{(0,0)\}$. We describe each of the considered vector fields $U_{0}$ in Lemma 3.4 and we note that they are, indeed, polynomial vector fields. Since $X_{0}$ and $U_{0}$ are transversal vector fields on $\mathcal{P}$, there exist two analytic functions $\lambda$ and $\mu$ such that

$$
Y_{\varepsilon}=X_{\varepsilon}-X_{0}=\lambda X_{0}+\mu U_{0}
$$

where $Y_{\varepsilon}$ is the vector field corresponding to the perturbative part as defined in (3). In fact it is clear that

$$
\lambda=\frac{Y_{\varepsilon} \wedge U_{0}}{X_{0} \wedge U_{0}} \quad \text { and } \quad \mu=\frac{X_{0} \wedge Y_{\varepsilon}}{X_{0} \wedge U_{0}} .
$$

Our first result, Theorem 3.2, gives an expression for the $(k+1)$ th term of the Taylor series of $T^{\prime}(s ; \varepsilon)$ at $\varepsilon=0$. It depends on the $(k+1)$ th term of the development of $\lambda=\lambda(x, y ; \varepsilon)$ at $\varepsilon=0$ and to prove it we shall use the following result (see [10]). This theorem is a generalization of the result stated in the seminal paper [9], see also the references therein.

Theorem 3.1 (Gasull-Yu). Let $X$ be an analytic vector field with a center at $p$ and let $\mathcal{P}$ be its period annulus. Let $U$ be an analytic vector field on $\mathcal{P} \cup\{p\}$ which is transversal to $X$ on $\mathcal{P}$. Then $[X, U]=\alpha X+\beta U$ for some analytic functions $\alpha$ and $\beta$. Fix any $q \in \mathcal{P}$ and let $\xi: \mathcal{I} \longrightarrow \mathcal{P}$ be the solution of $U$ with $\xi(0)=q$. For each $s \in \mathcal{I}$, let $\varphi(t ; s)$ be the solution of $X$ with $\varphi(0 ; s)=\xi(s)$ and let $T(s)$ be its period. Then

$$
T^{\prime}(s)=\int_{0}^{T(s)} \alpha(\varphi(t ; s)) \exp \left(-\int_{0}^{t} \beta(\varphi(\tau ; s)) d \tau\right) d t \text { for all } s \in \mathcal{I} .
$$

Let us point out that in the above statement $U$ is just a transversal vector field to $X$. We can now prove the following:

Theorem 3.2. Consider the parameterization of the period function $T(s ; \varepsilon)$ as introduced in Definition 2.3. Let us assume that, for some $k \in \mathbb{N}$, there exists an analytic family of diffeomorphisms $\left\{\Phi_{\varepsilon}\right\}$, in a neighbourhood of $(0,0)$, such that $\Phi_{\varepsilon}$ linearizes $j^{k}\left(X_{\varepsilon}\right)$ for each $\varepsilon \approx 0$. Then $T_{0}^{\prime} \equiv T_{1}^{\prime} \equiv \ldots \equiv T_{k}^{\prime} \equiv 0$ and

$$
T_{k+1}^{\prime}(s)=-\left.\int_{0}^{T_{0}} U_{0}\left(\lambda_{k+1}\right)\right|_{(x, y)=\varphi(t ; s)} d t \text { for all } s \in \mathcal{I},
$$

where $\varphi(t ; s)$ is the solution of $X_{0}$ with $\varphi(0 ; s)=\xi(s)$. 
Proof. We focus in the formula for $T_{k+1}^{\prime}$ because as a byproduct it will follow that $T_{0}^{\prime} \equiv T_{1}^{\prime} \equiv \ldots \equiv T_{k}^{\prime} \equiv 0$. Note first that it suffices to show the validity of the equality for periodic orbits near the origin since the functions on the left and right of the equality depend analytically on $s$. To this end we use the family of diffeomorphisms $\left\{\Phi_{\varepsilon}\right\}$ that are defined in neighbourhood of the origin. (Certainly this family depends on $k$ as well, but for the sake of shortness we omit this additional subscript.)

Define $\Psi_{\varepsilon}:=\left(\Phi_{0}\right)^{-1} \circ \Phi_{\varepsilon}$. Then $\left\{\Psi_{\varepsilon}\right\}$ is an analytic family of diffeomorphisms with $\Psi_{\varepsilon}=\operatorname{Id}+o\left(\varepsilon^{0}\right)$ and such that, for each $\varepsilon \approx 0, \Psi_{\varepsilon}$ conjugates $j^{k}\left(X_{\varepsilon}\right)$ and $X_{0}$, i.e., $\left(\Psi_{\varepsilon}\right)^{*}\left(j^{k}\left(X_{\varepsilon}\right)\right)=X_{0}$. Let us define in addition $U_{\varepsilon}:=\left(\Psi_{\varepsilon}\right)_{*}\left(U_{0}\right)$ and note that then $U_{\varepsilon}=U_{0}+\mathrm{o}\left(\varepsilon^{0}\right)$. We claim that

$$
\left[X_{\varepsilon}, U_{\varepsilon}\right]=\varepsilon^{k+1}\left(-U_{0}\left(\lambda_{k+1}\right)+o\left(\varepsilon^{0}\right)\right) X_{\varepsilon}+\varepsilon^{k+1}\left(-U_{0}\left(\mu_{k+1}\right)+o\left(\varepsilon^{0}\right)\right) U_{\varepsilon}
$$

where here (and in what follows) we use the notation $\lambda=\sum_{i=0}^{\infty} \lambda_{i} \varepsilon^{i}$ and $\mu=\sum_{i=0}^{\infty} \mu_{i} \varepsilon^{i}$. In order to prove the claim let us first recall that $X_{\varepsilon}=X_{0}+\lambda X_{0}+\mu U_{0}$. Thus, we can write

$$
X_{\varepsilon}=j^{k}\left(X_{\varepsilon}\right)+\varepsilon^{k+1}\left(\left(\lambda_{k+1}+\mathrm{o}\left(\varepsilon^{0}\right)\right) X_{0}+\left(\mu_{k+1}+\mathrm{o}\left(\varepsilon^{0}\right)\right) U_{0}\right) .
$$

On the other hand,

$$
\left(\Psi_{\varepsilon}\right)^{*}\left[j^{k}\left(X_{\varepsilon}\right), U_{\varepsilon}\right]=\left[\left(\Psi_{\varepsilon}\right)^{*}\left(j^{k}\left(X_{\varepsilon}\right)\right),\left(\Psi_{\varepsilon}\right)^{*}\left(U_{\varepsilon}\right)\right]=\left[X_{0},\left(\Psi_{\varepsilon}\right)^{*}\left(\left(\Psi_{\varepsilon}\right)_{*}\left(U_{0}\right)\right)\right]=\left[X_{0}, U_{0}\right]=0 .
$$

In the first equality above we use that if $f$ is a local diffeomorphism, then $f^{*}[X, Y]=\left[f^{*} X, f^{*} Y\right]$, see for instance [14. In the second one we use that $\Psi_{\varepsilon}$ conjugates $j^{k}\left(X_{\varepsilon}\right)$ and $X_{0}$ and that, by definition, $U_{\varepsilon}=\left(\Psi_{\varepsilon}\right)_{*}\left(U_{0}\right)$. The last one follows from the fact that $U_{0}$ is a commutator of $X_{0}$. We can thus conclude that $\left[j^{k}\left(X_{\varepsilon}\right), U_{\varepsilon}\right]=0$. Accordingly, from (7) we obtain

$$
\left[X_{\varepsilon}, U_{\varepsilon}\right]=\underbrace{\left[j^{k}\left(X_{\varepsilon}\right), U_{\varepsilon}\right]}_{0}+\varepsilon^{k+1} \underbrace{\left[\left(\lambda_{k+1}+o\left(\varepsilon^{0}\right)\right) X_{0}, U_{\varepsilon}\right]}_{\Delta_{1}}+\varepsilon^{k+1} \underbrace{\left[\left(\mu_{k+1}+o\left(\varepsilon^{0}\right)\right) U_{0}, U_{\varepsilon}\right]}_{\Delta_{2}} .
$$

Then, since $[f X, Y]=f[X, Y]-Y(f) X$ (see [14] again), it follows that

$$
\begin{aligned}
\Delta_{1} & =\left(\lambda_{k+1}+\mathrm{o}\left(\varepsilon^{0}\right)\right)\left[X_{0}, U_{\varepsilon}\right]-U_{\varepsilon}\left(\lambda_{k+1}+\mathrm{o}\left(\varepsilon^{0}\right)\right) X_{0} \\
& =\left(\lambda_{k+1}+\mathrm{o}\left(\varepsilon^{0}\right)\right)\left[X_{0}, U_{0}+\mathrm{o}\left(\varepsilon^{0}\right)\right]-U_{0}\left(\lambda_{k+1}\right) X_{\varepsilon}+\mathrm{o}\left(\varepsilon^{0}\right)=-U_{0}\left(\lambda_{k+1}\right) X_{\varepsilon}+\mathrm{o}\left(\varepsilon^{0}\right)
\end{aligned}
$$

and

$$
\Delta_{2}=\left(\mu_{k+1}+\mathrm{o}\left(\varepsilon^{0}\right)\right)\left[U_{0}, U_{\varepsilon}\right]-U_{\varepsilon}\left(\mu_{k+1}+\mathrm{o}\left(\varepsilon^{0}\right)\right) U_{0}=-U_{0}\left(\mu_{k+1}\right) U_{\varepsilon}+\mathrm{o}\left(\varepsilon^{0}\right),
$$

where we took $X_{\varepsilon}-X_{0}=U_{\varepsilon}-U_{0}=\mathrm{o}\left(\varepsilon^{0}\right)$ and $\left[X_{0}, U_{0}\right]=0$ into account. Hence

$$
\left[X_{\varepsilon}, U_{\varepsilon}\right]=\varepsilon^{k+1}\left(-U_{0}\left(\lambda_{k+1}\right)\right) X_{\varepsilon}+\varepsilon^{k+1}\left(-U_{0}\left(\mu_{k+1}\right)\right) U_{\varepsilon}+\mathrm{o}\left(\varepsilon^{k+1}\right),
$$

and (6) follows after decomposing the remainder term above in the $X_{\varepsilon}$ and $U_{\varepsilon}$ components using that both vector fields are transversal for $\varepsilon \approx 0$. This shows that the claim is true.

Next we take the point $q \in \mathcal{P}$ in Definition 2.3 and consider the solution $\psi_{\varepsilon}(s ; q)$ of $X_{\varepsilon}$ with $\psi_{\varepsilon}(0 ; q)=q$. If $\widehat{T}(s ; \varepsilon)$ denotes the period of the periodic orbit of $X_{\varepsilon}$ passing through $\xi_{\varepsilon}(s):=\psi_{\varepsilon}(s ; q)$, then from (6) and applying Theorem 3.1 it follows that

$$
\widehat{T}^{\prime}(s ; \varepsilon)=-\left.\varepsilon^{k+1} \int_{0}^{\widehat{T}(s ; \varepsilon)}\left(U_{0}\left(\lambda_{k+1}\right)+\mathrm{o}\left(\varepsilon^{0}\right)\right)\right|_{(x, y)=\varphi_{\varepsilon}(t ; s)} e^{-\left.\varepsilon^{k+1} \int_{0}^{t}\left(U_{\varepsilon}\left(\mu_{k+1}\right)+\mathrm{O}\left(\varepsilon^{0}\right)\right)\right|_{(x, y)=\varphi \varepsilon(\tau ; s)} d \tau} d t,
$$

where $\varphi_{\varepsilon}(t ; s)$ is the solution of $X_{\varepsilon}$ with $\varphi_{\varepsilon}(0 ; s)=\xi_{\varepsilon}(s)$. Therefore, on account of $X_{\varepsilon}-X_{0}=U_{\varepsilon}-U_{0}=o\left(\varepsilon^{0}\right)$, we obtain

$$
\widehat{T}^{\prime}(s ; \varepsilon)=-\left.\varepsilon^{k+1} \int_{0}^{T_{0}} U_{0}\left(\lambda_{k+1}\right)\right|_{(x, y)=\varphi_{0}(t ; s)} d t+o\left(\varepsilon^{k+1}\right) .
$$


Note at this point that, by construction, $T(s ; \varepsilon)=\widehat{T}(f(s, \varepsilon) ; \varepsilon)$ for some function $f$ with $f(s, 0)=s$. Taking this into account, one can easily show from the above equality that

$$
T^{\prime}(s ; \varepsilon)=-\left.\varepsilon^{k+1} \int_{0}^{T_{0}} U_{0}\left(\lambda_{k+1}\right)\right|_{(x, y)=\varphi_{0}(t ; s)} d t+o\left(\varepsilon^{k+1}\right) .
$$

Accordingly, $T_{0}^{\prime} \equiv T_{1}^{\prime} \equiv \ldots \equiv T_{k}^{\prime} \equiv 0$ and

$$
T_{k+1}^{\prime}(s)=-\left.\int_{0}^{T_{0}} U_{0}\left(\lambda_{k+1}\right)\right|_{(x, y)=\varphi(t ; s)} d t .
$$

This completes the proof of the result.

Remark 3.3 As we explain at the end of Section 2. it is well known that a vector field has an isochronous center if and only if it is linearizable. The authors do not know weather this characterization is true for families. This is the reason why the assumption in Theorem 3.2 requires the existence of an analytic family of linearizations instead of assuming that the center of $j^{k}\left(X_{\varepsilon}\right)$ is isochronous for $\varepsilon \approx 0$. The latter assumption provides a linearization $\Phi_{\varepsilon}$ for each $\varepsilon \approx 0$, but in general we know nothing about the regularity of the family $\left\{\Phi_{\varepsilon}\right\}$ with respect to $\varepsilon$.

The following lemma (see [5, 21) provides the necessary information in order to apply the above result to our problem with the Pleshkan's isochrones. It gives the commutator and first integral of each isochronous center in (2).

Lemma 3.4. $\left(S_{1}^{*}\right)$ The vector field $X_{0}=\left(-y-3 x^{2} y+y^{3}\right) \partial_{x}+\left(x+x^{3}-3 x y^{2}\right) \partial_{y}$ has first integral

$$
H(x, y)=\frac{x^{2}+y^{2}}{\sqrt{\left(x^{2}+(y-1)^{2}\right)\left(x^{2}+(y+1)^{2}\right)}}
$$

and commutator $U_{0}=\left(x+x^{3}-3 x y^{2}\right) \partial_{x}+\left(y+3 x^{2} y-y^{3}\right) \partial_{y}$.

$\left(S_{2}^{*}\right)$ The vector field $X_{0}=\left(-y+x^{2} y\right) \partial_{x}+\left(x+x y^{2}\right) \partial_{y}$ has first integral

$$
H(x, y)=\frac{x^{2}+y^{2}}{1-x^{2}}
$$

and commutator $U_{0}=x\left(1-x^{2}\right) \partial_{x}+y\left(1-x^{2}\right) \partial_{y}$.

$\left(S_{3}^{*}\right)$ The vector field $X_{0}=\left(-y+3 x^{2} y\right) \partial_{x}+\left(x-2 x^{3}+9 x y^{2}\right) \partial_{y}$ has first integral

$$
H(x, y)=\frac{\left(x-2 x^{3}\right)^{2}+y^{2}}{\left(1-3 x^{2}\right)^{3}}
$$

and commutator $U_{0}=x\left(1-3 x^{2}\right)\left(1-2 x^{2}\right) \partial_{x}+y\left(1-3 x^{2}\right)\left(1-6 x^{2}\right) \partial_{y}$.

$\left(\bar{S}_{3}^{*}\right)$ The vector field $X_{0}=\left(-y-3 x^{2} y\right) \partial_{x}+\left(x+2 x^{3}-9 x y^{2}\right) \partial_{y}$ has first integral

$$
H(x, y)=\frac{\left(x+2 x^{3}\right)^{2}+y^{2}}{\left(1+3 x^{2}\right)^{3}}
$$

and commutator $U_{0}=x\left(1+3 x^{2}\right)\left(1+2 x^{2}\right) \partial_{x}+y\left(1+3 x^{2}\right)\left(1+6 x^{2}\right) \partial_{y}$.

Since the number of critical periods that bifurcate from $\mathcal{P}$ does not depend on the particular parameterization of the set of periodic orbits in $\mathcal{P}$ used (recall Remark 2.2 ), Theorem $\mathrm{A}$ will follow once we prove the next result. 
Theorem 3.5. Let $X_{0}$ be one of the four Pleshkan's isochrones in Lemma 3.4 and let $U_{0}$ be its corresponding commutator. Consider the unfolding of centers $X_{\varepsilon}:=X_{0}+Y_{\varepsilon}$ where

$$
Y_{\varepsilon}=\left(a(\varepsilon) x^{2} y+b(\varepsilon) y^{3}\right) \partial_{x}+\left(c(\varepsilon) x^{3}+d(\varepsilon) x y^{2}\right) \partial_{y}
$$

and where $a, b, c$ and $d$ are analytic functions in a neighbourhood of $\varepsilon=0$ with $a(0)=b(0)=c(0)=d(0)=0$. Let $\xi: \mathcal{I} \longrightarrow \mathbb{R}^{2}$ be a transversal section to $\mathcal{P}$ given by a solution of $U_{0}$ and let $T(s ; \varepsilon)$ be the period of the periodic orbit of $X_{\varepsilon}$ passing through $\xi(s) \in \mathcal{P}$. If $T_{0}^{\prime} \equiv T_{1}^{\prime} \equiv \ldots T_{k}^{\prime} \equiv 0$ and $T_{k+1}^{\prime} \not \equiv 0$, then $T_{k+1}^{\prime}(s)$ has at most two zeros for $s \in \mathcal{I}$ and there are perturbations $Y_{\varepsilon}$ giving rise to 0,1 and 2 zeros.

In order to prove this result some definitions and lemmas are needed.

Definition 3.6 Let $f_{0}, f_{1}, \ldots, f_{n-1}$ be analytic functions on an open interval $L$ of $\mathbb{R}$.

(a) $\left(f_{0}, f_{1}, \ldots, f_{n-1}\right)$ is a Chebyshev system (in short, T-system) on $L$ if any nontrivial linear combination

$$
\alpha_{0} f_{0}(x)+\alpha_{1} f_{1}(x)+\ldots+\alpha_{n-1} f_{n-1}(x)
$$

has at most $n-1$ isolated zeros on $L$.

(b) $\left(f_{0}, f_{1}, \ldots, f_{n-1}\right)$ is a complete Chebyshev system (in short, CT-system) on $L$ if $\left(f_{0}, f_{1}, \ldots, f_{k-1}\right)$ is a T-system for all $k=1,2, \ldots, n$.

(c) $\left(f_{0}, f_{1}, \ldots, f_{n-1}\right)$ is an extended complete Chebyshev system (in short, ECT-system) on $L$ if, for all $k=1,2, \ldots, n$, any nontrivial linear combination

$$
\alpha_{0} f_{0}(x)+\alpha_{1} f_{1}(x)+\ldots+\alpha_{k-1} f_{k-1}(x)
$$

has at most $k-1$ isolated zeros on $L$ counted with multiplicities.

(Let us mention that, in these abbreviations, "T" stands for Tchebycheff, which in some sources is the transcription of the Russian name Chebyshev.)

Remark 3.7 If $\left(f_{0}, f_{1}, \ldots, f_{n-1}\right)$ is an ECT-system on $L$, then for each $k=0,1, \ldots, n-1$ there exists a linear combination

$$
\alpha_{0} f_{0}(x)+\alpha_{1} f_{1}(x)+\ldots+\alpha_{n-1} f_{n-1}(x)
$$

with exactly $k$ simple zeros on $L$ (see for instance [13, 17]).

Definition 3.8 Let $f_{0}, f_{1}, \ldots, f_{k-1}$ be analytic functions on an open interval $L$ of $\mathbb{R}$. The Wronskian of $\left(f_{0}, f_{1}, \ldots, f_{k-1}\right)$ at $x \in L$ is

$$
W\left[f_{0}, f_{1}, \cdots, f_{k-1}\right](x)=\operatorname{det}\left(f_{j}^{(i)}(x)\right)_{0 \leqslant i, j \leqslant k-1}=\left|\begin{array}{rrr}
f_{0}(x) & \cdots & f_{k-1}(x) \\
f_{0}^{\prime}(x) & \cdots & f_{k-1}^{\prime}(x) \\
& \vdots & \\
f_{0}^{(k-1)}(x) & \cdots & f_{k-1}^{(k-1)}(x)
\end{array}\right|
$$

The following result is well known (see [13, 17] for instance).

Lemma 3.9. $\left(f_{0}, f_{1}, \ldots, f_{n-1}\right)$ is an ECT-system on $L$ if, and only if, for each $k=1,2, \ldots, n$ it holds

$$
W\left[f_{0}, f_{1}, \cdots, f_{k-1}\right](x) \neq 0 \text { for all } x \in L .
$$


Recall that Theorem 3.5 deals with four different isochrones. For each one, the proof yields to a linear combination of some Abelian integrals and it is necessary to show that they form an ECT-system. This is in general extremely complicated to verify, but in three of the cases it is not that difficult because a criterion proved in 12 applies successfully. Proposition 3.10 is a simplified version of this criterion that it suffices for our purposes. In its statement we suppose that the projection of $\mathcal{P}$ on the $x$-axis is given by $\left(-x_{r}, x_{r}\right)$ and that the energy level of $H$ at the periodic orbits in $\mathcal{P}$ ranges from $h=0$ to $h=h_{0}$.

Proposition 3.10. Let us consider the Abelian integrals

$$
I_{i}(h)=\int_{\gamma_{h}} f_{i}(x) y^{2 m-1} d x, \quad i=0,1, \ldots, n-1,
$$

where $f_{i}(x)$ are analytic functions in $\left(-x_{r}, x_{r}\right), m \in \mathbb{Z}$ and where, for each $h \in\left(0, h_{0}\right)$, $\gamma_{h}$ is the oval surrounding the origin inside the level curve $\left\{A(x)+B(x) y^{2}=h\right\}$. Assume that $A$ and $B$ are even functions and let $\ell_{i}$ be the even part of $f_{i}$. Then $\left(I_{0}, I_{1}, \ldots, I_{n-1}\right)$ is an ECT-system on $\left(0, h_{0}\right)$ if $m \geqslant n-1$ and $\left(\ell_{0}, \ell_{1}, \ldots, \ell_{n-1}\right)$ is a CT-system on $\left(0, x_{r}\right)$.

The following lemma, proved in [12, establishes a formula to write the integrand of an Abelian integral so as to be suitable to apply the Proposition 3.10 .

Lemma 3.11. Let $\gamma_{h}$ be the oval surrounding the origin inside the level curve $\left\{A(x)+B(x) y^{2}=h\right\}$ and we consider a function $F$ such that $F / A^{\prime}$ is analytic at $x=0$. Then, for any $k \in \mathbb{N}$,

$$
\int_{\gamma_{h}} F(x) y^{k-2} d x=\int_{\gamma_{h}} G(x) y^{k} d x
$$

where $G(x)=\frac{2}{k}\left(\frac{B F}{A^{\prime}}\right)^{\prime}(x)-\left(\frac{B^{\prime} F}{A^{\prime}}\right)(x)$.

The criterion in Proposition 3.10 does not apply for the isochronous center $\left(S_{1}^{*}\right)$. This fact makes the proof of Theorem 3.5 for this case much longer than the others. In particular we shall need some properties of the complete elliptic integrals (see [1] for instance).

Definition 3.12 The complete elliptic integrals of first and second kind are

$$
\mathcal{E}(u):=\int_{0}^{\pi / 2} \sqrt{1-u^{2} \sin ^{2} t} d t \text { and } \mathcal{K}(u):=\int_{0}^{\pi / 2} \frac{d t}{\sqrt{1-u^{2} \sin ^{2} t}}
$$

respectively, which are analytic real functions for $u \in(-1,1)$.

Lemma 3.13. The functions $\mathcal{K}$ and $\mathcal{E}$ verify the linear differential equation

$$
\left(\begin{array}{c}
\mathcal{K}^{\prime} \\
\mathcal{E}^{\prime}
\end{array}\right)=\frac{-1}{u}\left(\begin{array}{cc}
1 & \frac{1}{u^{2}-1} \\
1 & -1
\end{array}\right)\left(\begin{array}{c}
\mathcal{K} \\
\mathcal{E}
\end{array}\right)
$$

Moreover, their Taylor series at $u=0$ are

$$
\mathcal{K}(u)=\frac{\pi}{2} \sum_{i \geqslant 0}\left(\frac{(2 i-1) ! !}{(2 i) ! !}\right)^{2} u^{2 i} \text { and } \mathcal{E}(u)=-\frac{\pi}{2} \sum_{i \geqslant 0}\left(\frac{(2 i-1) ! !}{(2 i) ! !}\right)^{2} \frac{u^{2 i}}{2 i-1}
$$

where $u \in(-1,1)$.

Recall that the double factorial of an integer $n$, with $n \geqslant-1$, is defined as

$$
n ! != \begin{cases}n(n-2) \ldots 5 \cdot 3 \cdot 1 & \text { if } n>0 \text { and } n \text { odd } \\ n(n-2) \ldots 6 \cdot 4 \cdot 2 & \text { if } n>0 \text { and } n \text { even } \\ 1 & \text { if } n=-1,0\end{cases}
$$

Finally, once we show the following lemma we will be in position to prove Theorem 3.5 . 
Lemma 3.14. Following the notation in the statement of Theorem 3.5, let us write $X_{\varepsilon}-X_{0}=\lambda X_{0}+\mu U_{0}$ and, setting $\lambda=\sum_{i=1}^{\infty} \lambda_{i} \varepsilon^{i}$, define

$$
R(s)=\left.\int_{0}^{T_{0}} U_{0}\left(\lambda_{k}\right)\right|_{(x, y)=\varphi(t ; s)} d t,
$$

where $\varphi(t ; s)$ is the solution of $X_{0}$ with $\varphi(0 ; s)=\xi(s)$. Then $R(s)=\alpha I_{0}(s)+\beta I_{1}(s)+\gamma I_{2}(s)$ where $\left(I_{0}, I_{1}, I_{2}\right)$ is an ECT-system on $\mathcal{I}$ and $(\alpha, \beta, \gamma)=\phi\left(a_{k}, b_{k}, c_{k}, d_{k}\right)$ with $\phi$ being a surjective linear mapping such that

(a) $\operatorname{Ker}(\phi)=\left\{d_{k}+3 c_{k}=a_{k}+3 c_{k}=b_{k}-c_{k}=0\right\}$ in case that $X_{0}$ is $\left(S_{1}^{*}\right)$,

(b) $\operatorname{Ker}(\phi)=\left\{b_{k}=c_{k}=a_{k}-d_{k}=0\right\}$ in case that $X_{0}$ is $\left(S_{2}^{*}\right)$,

(c) $\operatorname{Ker}(\phi)=\left\{b_{k}=2 a_{k}+3 c_{k}=2 d_{k}+9 c_{k}=0\right\}$ in case that $X_{0}$ is $\left(S_{3}^{*}\right)$ or $\left(\bar{S}_{3}^{*}\right)$.

Proof. First we shall write $R(s)$ as an Abelian integral taking advantage of the fact that each isochronous center $X_{0}$ has a first integral $H$, see Lemma 3.4 Thus, if $\gamma_{s}$ denotes the periodic orbit of $X_{0}$ inside the energy level $\{H(x, y)=H(\xi(s))\}$ and $X_{0}=P_{0} \partial_{x}+Q_{0} \partial_{y}$, then

$$
R(s)=\int_{\gamma_{s}} \frac{<\nabla \lambda_{k}(x, y), U_{0}(x, y)>}{P_{0}(x, y)} d x,
$$

where $<,>$ denotes the inner product. In fact, setting $\eta(s):=H(\xi(s))$, for simplicity in the exposition we shall study

$$
I(h):=R\left(\eta^{-1}(h)\right)=\int_{\gamma_{h}} \frac{<\nabla \lambda_{k}(x, y), U_{0}(x, y)>}{P_{0}(x, y)} d x,
$$

where $\gamma_{h}$ is now the oval inside the level curve $\{H(x, y)=h\}$. Note moreover that, by definition, $Y_{\varepsilon}=X_{\varepsilon}-X_{0}$ and $X_{\varepsilon}-X_{0}=\lambda X_{0}+\mu U_{0}$, so that

$$
\lambda=\frac{Y_{\varepsilon} \wedge U_{0}}{X_{0} \wedge U_{0}} .
$$

We are now in position to prove the result for each different case.

The case $\left(\mathbf{S}_{\mathbf{2}}^{*}\right)$. From the above equality, taking the expression of $X_{0}$ and $U_{0}$ given by Lemma 3.4 into account, an easy computation shows that

$$
\lambda(x, y ; \varepsilon)=-\frac{a(\varepsilon) y^{2} x^{2}+b(\varepsilon) y^{4}-c(\varepsilon) x^{4}-d(\varepsilon) x^{2} y^{2}}{x^{2}+y^{2}},
$$

and accordingly, since $\lambda=\sum_{i=1}^{\infty} \lambda_{i} \varepsilon^{i}$, it turns out that

$$
\lambda_{k}(x, y)=-\frac{a_{k} y^{2} x^{2}+b_{k} y^{4}-c_{k} x^{4}-d_{k} x^{2} y^{2}}{x^{2}+y^{2}} .
$$

From now on, when there is no risk of ambiguity, we shall omit the subscript $k$ for the sake of shortness. In fact it does not play any role at all because $\lambda_{k}(x, y)=F\left(a_{k}, b_{k}, c_{k}, d_{k}, x, y\right)$ with $F$ not depending on $k$. Since $H(x, y)=\frac{x^{2}+y^{2}}{1-x^{2}}$, the projection of $\mathcal{P}$ on the $x$-axis is $(-1,1)$ and the energy level in $\gamma_{h}$ ranges from $h=0$ to $h_{0}=+\infty$. It turns out that

$$
I(h)=2 \int_{\gamma_{h}} \frac{b y^{4}+(a-d) x^{2} y^{2}-c x^{4}}{y\left(x^{2}+y^{2}\right)} d x=\frac{2}{h} \int_{\gamma_{h}} \frac{b y^{4}+(a-d) x^{2} y^{2}-c x^{4}}{y\left(1-x^{2}\right)} d x,
$$

where in the last equality we use that $x^{2}+y^{2}=\left(1-x^{2}\right) h$. Consequently

$$
I(h)=\frac{2}{h}\left(b I_{0}(h)+(a-d) I_{1}(h)-c I_{2}(h)\right)
$$


with

$$
I_{0}(h)=\int_{\gamma_{h}} \frac{y^{3} d x}{1-x^{2}}, I_{1}(h)=\int_{\gamma_{h}} \frac{x^{2} y d x}{1-x^{2}} \text { and } I_{2}(h)=\int_{\gamma_{h}} \frac{x^{4} d x}{y\left(1-x^{2}\right)} .
$$

Clearly, since $\eta(s)=H(\xi(s))$ is a diffeomorphism and $\eta(\mathcal{I})=(0,+\infty)$, setting $\phi(a, b, c, d)=(b, a-d,-c)$, the proof of Lemma 3.14 will follow for this case once we show that $\left(I_{0}, I_{1}, I_{2}\right)$ is an ECT-system on $(0,+\infty)$. To this end we shall apply Proposition 3.10. With this aim in view, the application of Lemma 3.11 to $I_{1}(h)$ with $k=3$ gives

$$
I_{1}(h)=\int_{\gamma_{h}} f_{1}(x) y^{3} d x \text { where } f_{1}(x)=\frac{1-4 x^{2}}{3\left(1-x^{2}\right)} .
$$

Similarly, but now applying twice Lemma 3.11, first with $k=1$ and then with $k=3$, we obtain

$$
I_{2}(h)=\int_{\gamma_{h}} f_{2}(x) y^{3} d x \text { where } f_{2}(x)=\frac{8 x^{4}-8 x^{2}+1}{1-x^{2}} .
$$

On the other hand, note that

$$
I_{0}(h)=\int_{\gamma_{h}} f_{0}(x) y^{3} d x \text { where } f_{0}(x)=\frac{1}{1-x^{2}} .
$$

Our goal with these manipulations is twofold. Firstly, we want that $y$ has the same exponent in all the Abelian integrals. Secondly, that this exponent is large enough so that, with the notation in Proposition 3.10 . $m \geqslant n-1$. Now, by applying this result, if $\left(f_{0}, f_{1}, f_{2}\right)$ is a CT-system on $(0,1)$, then $\left(I_{0}, I_{1}, I_{2}\right)$ is an ECTsystem on $(0,+\infty)$, and we are done. This is clear because $f_{0}, f_{1}$ and $f_{2}$ share the same denominator and each numerator is an even polynomial of degree exactly $2 i$ for $\mathrm{i}=0,1,2$.

The case $\left(\mathbf{S}_{\mathbf{3}}^{*}\right)$. We omit many of the explanations for the sake of brevity because the proof in this case is completely analogous to the previous one. Now the Abelian integral $I(h)=R\left(\eta^{-1}(h)\right)$ is given by

$$
I(h)=\frac{2}{h} \int_{\gamma_{h}}\left(\frac{c x^{4}\left(1-2 x^{2}\right)}{\left(1-3 x^{2}\right)^{3} y}+\frac{x^{2}\left(1-2 x^{2}\right)\left(d-a+4 x^{2}(3 a-d)\right) y}{\left(1-3 x^{2}\right)^{3}}-\frac{b\left(1-18 x^{2}+48 x^{4}\right) y^{3}}{\left(1-3 x^{2}\right)^{3}}\right) d x,
$$

where we used the transversal commutator and the first integral given by Lemma 3.4 The projection of $\mathcal{P}$ on the $x$-axis is $\left(-\frac{1}{\sqrt{3}}, \frac{1}{\sqrt{3}}\right)$ and $h_{0}=+\infty$. By applying Lemma 3.11 exactly as in the previous case we obtain

$$
I(h)=\frac{2}{3 h}\left((d-a-3 b+3 c) I_{0}(h)+6(9 a+9 b-9 c-5 d) I_{1}(h)-48(6 a+3 b-2 d) I_{2}(h)\right),
$$

where

$$
I_{i}(h)=\int_{\gamma_{h}} f_{i}(x) y^{3} d x \text { with } f_{i}(x)=\frac{x^{2 i}}{\left(1-3 x^{2}\right)^{3}} \text { for } i=0,1,2 .
$$

By applying Proposition 3.10, $\left(I_{0}, I_{1}, I_{2}\right)$ is an ECT-system on $(0,+\infty)$ because it is clear that $\left(f_{0}, f_{1}, f_{2}\right)$ is a CT-system on $\left(0, \frac{1}{\sqrt{3}}\right)$. Thus, taking $\phi(a, b, c, d)=(d-a-3 b+3 c, 9 a+9 b-9 c-5 d, 6 a+3 b-2 d)$, the result follows in this case.

The case $\left(\overline{\mathbf{S}}_{\mathbf{3}}^{*}\right)$. Exactly in the same way as in the previous cases, the Abelian integral $I(h)=R\left(\eta^{-1}(h)\right)$ is given by

$$
I(h)=\frac{2}{h} \int_{\gamma_{h}}\left(\frac{c x^{4}\left(1+2 x^{2}\right)}{\left(1+3 x^{2}\right)^{3} y}+\frac{x^{2}\left(1+2 x^{2}\right)\left(d-a+4 x^{2}(d-3 a)\right) y}{\left(1+3 x^{2}\right)^{3}}-\frac{b\left(1+18 x^{2}+48 x^{4}\right) y^{3}}{\left(1+3 x^{2}\right)^{3}}\right) d x .
$$

The projection of the period annulus $\mathcal{P}$ on the $x$-axis is $(-\infty,+\infty)$ and $h_{0}=\frac{4}{27}$. By applying Lemma 3.11.

$$
I(h)=\frac{2}{3 h}\left((d-a-3 b+3 c) I_{0}(h)-6(9 a+9 b-9 c-5 d) I_{1}(h)-48(6 a+3 b-2 d) I_{2}(h)\right),
$$


where

$$
I_{i}(h)=\int_{\gamma_{h}} f_{i}(x) y^{3} d x \text { with } f_{i}(x)=\frac{x^{2 i}}{\left(1+3 x^{2}\right)^{3}} \text { for } i=0,1,2 .
$$

We are under the hypothesis of Proposition 3.10 and, since it is obvious that $\left(f_{0}, f_{1}, f_{2}\right)$ is a CT-system on $(0,+\infty)$, we conclude that $\left(I_{0}, I_{1}, I_{2}\right)$ is an ECT-system on $\left(0, \frac{4}{27}\right)$. Consequently the result follows taking, as before, $\phi(a, b, c, d)=(d-a-3 b+3 c, 9 a+9 b-9 c-5 d, 6 a+3 b-2 d)$.

The case $\left(\mathbf{S}_{1}^{*}\right)$. The proof for the perturbation of this isochronous center is longer and more complicated than the others because the condition for the first integral to take the form $A(x)+B(x) y^{2}$, as established in Proposition 3.10, is not verified. Then we must apply Lemma 3.9, that characterizes the Chebyshev property in terms of Wronskians. For this reason, instead of introducing Abelian integrals, we keep the expression of $R(s)$ in terms of the solution of the unperturbed system and, for the sake of convenience, we use complex notation. Recall that we now study the unfolding

$$
\left\{\begin{array}{l}
\dot{x}=-y+(-3+a(\varepsilon)) x^{2} y+(1+b(\varepsilon)) y^{3}, \\
\dot{y}=x+(1+c(\varepsilon)) x^{3}+(-3+d(\varepsilon)) x y^{2} .
\end{array}\right.
$$

Taking the commutator given by Lemma 3.4, an easy computation shows that

$$
\lambda(x, y ; \varepsilon)=\frac{x^{2}\left(1+x^{2}-3 y^{2}\right)\left(c(\varepsilon) x^{2}+d(\varepsilon) y^{2}\right)-y^{2}\left(1+3 x^{2}-y^{2}\right)\left(a(\varepsilon) x^{2}+b(\varepsilon) y^{2}\right)}{\left(x^{2}+y^{2}\right)\left(1+2\left(x^{2}-y^{2}\right)+\left(x^{2}+y^{2}\right)^{2}\right)} .
$$

The coordinate transformation $z=x+i y$ brings the unfolding to $\dot{z}=f(z)+i p(z, \bar{z})$ with

$$
f(z)=i z\left(1+z^{2}\right) \quad \text { and } \quad p(z, \bar{z})=\left(\alpha z^{3}+\beta z^{2} \bar{z}+\gamma z \bar{z}^{2}+\delta \bar{z}^{3}\right) / 8
$$

where $\alpha=b+c-d-a, \beta=3 c+d-a-3 b, \gamma=a+3 b+3 c+d$ and $\delta=a-b+c-d$. An easy computation shows that the solution of the unperturbed system, i.e., $\dot{z}=f(z)$, with initial condition $z=h \in(0,+\infty)$ at $t=0$ is given by

$$
\varphi(t ; h)=\frac{h e^{i t}}{\sqrt{1+h^{2}-e^{2 i t} h^{2}}} .
$$

Accordingly, following the usual notation $\alpha(\varepsilon)=\sum_{i=1}^{\infty} \alpha_{i} \varepsilon^{i}$, we split the function under consideration as

$$
R(h)=\int_{0}^{2 \pi}<\nabla \lambda_{k}(x, y), U_{0}(x, y)>\left.\right|_{x+i y=\varphi(t ; h)} d t=\alpha_{k} I_{3}(h)+\beta_{k} I_{2}(h)+\gamma_{k} I_{1}(h)+\delta_{k} I_{0}(h) .
$$

Long but straightforward manipulations show that $I_{3} \equiv 0$ and that

$$
R(h)=\frac{-4 h^{4}}{\left(1+h^{2}\right)^{2}}\left(\beta_{k} \bar{I}_{2}(h)+\gamma_{k} \pi+\delta_{k} \bar{I}_{0}(h)\right)
$$

where, setting $\mu(h)=\frac{2 h \sqrt{1+h^{2}}}{1+2 h^{2}}$,

$$
\bar{I}_{2}(h):=\frac{2 h^{2}}{1+2 h^{2}} \mathcal{K}(\mu(h))-\frac{2+4 h^{2}}{h^{2}} \mathcal{E}(\mu(h))
$$

and

$$
\bar{I}_{0}(h):=\frac{\left(1+2 h^{2}\right)\left(1+2 h^{2}+2 h^{4}\right)}{h^{6}} \mathcal{E}(\mu(h))-\frac{\left(1+h^{2}+h^{4}\right)\left(1+3 h^{2}+3 h^{4}\right)}{h^{6}\left(1+2 h^{2}\right)} \mathcal{K}(\mu(h)) .
$$

The fact that $I_{3} \equiv 0$ is not unexpected at all. If $\beta=\gamma=\delta=0$, then the perturbed system is holomorphic, it does not depend on $\bar{z}$, and consequently the center is isochronous for all $\varepsilon$, so that $T^{\prime}(s ; \varepsilon) \equiv 0$, see 25 . 
At this point, taking $\phi(a, b, c, d)=(3 c+d-a-3 b, a+3 b+3 c+d, a-b+c-d)$, the proof of Lemma 3.14 for the case $\left(S_{1}^{*}\right)$ reduces to the verification of the fact that $\left(\pi, \bar{I}_{2}, \bar{I}_{0}\right)$ is an ECT-system on $(0,+\infty)$. Since the first function is a non-zero constant, we compute the two-dimensional Wronskian by using Lemma 3.13 .

$$
W\left[\pi, \bar{I}_{2}\right](h)=\bar{I}_{2}^{\prime}(h)=\frac{2+2 h^{2}}{h^{3}}\left(\mathcal{E}(\mu(h))+\frac{\mathcal{K}(\mu(h))}{1+2 h^{2}}\right)
$$

Clearly it is different from zero for all $h \in(0,+\infty)$ because $\mathcal{E}$ and $\mathcal{K}$ are strictly positive functions. Next, taking Lemma 3.13 into account again, we compute the three-dimensional Wronskian. The key point is that it factorizes as

$$
W\left[\pi, \bar{I}_{2}, \bar{I}_{0}\right](h)=\left|\begin{array}{cc}
\bar{I}_{2}^{\prime} & \bar{I}_{0}^{\prime} \\
\bar{I}_{2}^{\prime \prime} & \bar{I}_{0}^{\prime \prime}
\end{array}\right|=\frac{72\left(1+h^{2}\right)^{3}}{h^{11}}\left(\mathcal{E}(\mu(h))+L_{+}(h) \mathcal{K}(\mu(h))\right)\left(\mathcal{E}(\mu(h))+L_{-}(h) \mathcal{K}(\mu(h))\right)
$$

where

$$
L_{ \pm}(h):=\frac{-1-2 h^{2}-2 h^{4} \pm 2\left(1+4 h^{2}+5 h^{4}+2 h^{6}+h^{8}\right)^{1 / 2}}{3\left(1+2 h^{2}\right)^{2}} .
$$

Note that $L_{-}(h)<0$ for all $h \in(0,+\infty)$. It is also easy to show that $L_{+}(h)>0$ for all $h \in(0,+\infty)$. Therefore, we will see that this Wronskian does not vanish once we prove that

$$
\mathcal{R}(h):=\mathcal{E}(\mu(h))+L_{-}(h) \mathcal{K}(\mu(h)) \neq 0 \text { for all } h \in(0,+\infty)
$$

From now on we will use the variable $u=\mu(h)$ because then the expressions that we obtain are shorter. Thus, due to $\mu(0,+\infty)=(0,1)$, we must show that

$$
\mathcal{L}(u):=\mathcal{R}\left(\mu^{-1}(u)\right)=\mathcal{E}(u)+\frac{1}{6}\left(u^{2}-2-\sqrt{16-16 u^{2}+u^{4}}\right) \mathcal{K}(u)
$$

does not vanish for all $u \in(0,1)$. To prove this claim we first note that by applying Lemma 3.13 one can check that $\mathcal{L}(u)$ verifies the differential equation

$$
\mathcal{L}^{\prime \prime}(u)=g_{1}(u) \mathcal{L}^{\prime}(u)+g_{0}(u) \mathcal{L}(u),
$$

where

$$
g_{1}(u)=\frac{48-64 u^{2}+17 u^{4}}{u\left(1-u^{2}\right)\left(16-16 u^{2}+u^{4}\right)}+\frac{\sqrt{16-16 u^{2}+u^{4}}}{u\left(1-u^{2}\right)}
$$

and

$$
g_{0}(u)=\frac{16-12 u^{2}+\left(8-u^{2}\right) \sqrt{16-16 u^{2}+u^{4}}}{\left(1-u^{2}\right)\left(16-16 u^{2}+u^{4}\right)} .
$$

On the other hand, from Lemma 3.13 once again, we get that $\mathcal{L}(u)=-\frac{3 \pi}{4096} u^{8}+\mathrm{o}\left(u^{9}\right)$. We are now in position to prove the claim. By contradiction, assume that there exists some $u_{1} \in(0,1)$ such that $\mathcal{L}\left(u_{1}\right)=0$. Then, since $\mathcal{L}(u)<0$ for $u \approx 0$, this forces the existence of a local minimum of $\mathcal{L}$, say $u_{0}$, with $\mathcal{L}\left(u_{0}\right)<0$. The evaluation of the differential equation in (8) at $u=u_{0}$ shows that $\mathcal{L}^{\prime \prime}\left(u_{0}\right)=g_{0}\left(u_{0}\right) \mathcal{L}\left(u_{0}\right)$. Since $g_{0}(u)>0$ for all $u \in(0,1)$, this implies that $\mathcal{L}^{\prime \prime}\left(u_{0}\right)<0$, which contradicts the fact that $u=u_{0}$ is a local minimum. Hence the claim is true and, therefore, the three-dimensional Wronskian is different from zero. In short, $\left(\pi, \bar{I}_{2}, \bar{I}_{0}\right)$ is an ECT-system on $(0,+\infty)$ and this completes the proof of the result for the case $\left(S_{1}^{*}\right)$. 
Proof of Theorem 3.5 We prove the result for the perturbation of the isochronous center $\left(S_{1}^{*}\right)$ only because the other cases follow exactly in the same way. Thus, consider the vector field $X_{0}$ with the isochronous center $\left(S_{1}^{*}\right)$ at the origin and, see Lemma 3.4. let $U_{0}$ be its commutator. In addition, let $\Phi$ be a linearization of $X_{0}$. As usual we consider the family of centers $X_{\varepsilon}=X_{0}+Y_{\varepsilon}$ and we denote by $T(s ; \varepsilon)$ the period function of $X_{\varepsilon}$ using a solution of $U_{0}$ as transversal section.

We claim that if $T_{0}^{\prime} \equiv T_{1}^{\prime} \equiv \ldots \equiv T_{k}^{\prime} \equiv 0$, then there exists an analytic family of diffeomorphisms $\left\{\Phi_{\varepsilon}^{k}\right\}$, in a neighbourhood of the origin, such that $\Phi_{\varepsilon}^{k}$ linearizes $j^{k}\left(X_{\varepsilon}\right)$ for all $\varepsilon \approx 0$. The proof follows by induction on $k$. The case $k=0$ is trivial because $j^{0}\left(X_{\varepsilon}\right)=X_{0}$ and so we can take $\Phi_{\varepsilon}^{0}=\Phi$. Assume that the claim is true for $k=n$ and let us show its validity for $k=n+1$. So suppose that $T_{0}^{\prime} \equiv T_{1}^{\prime} \equiv \ldots \equiv T_{n}^{\prime} \equiv T_{n+1}^{\prime} \equiv 0$ and, by the induction hypothesis, that there exists a linearization $\Phi_{\varepsilon}^{k}$ of $j^{k}\left(X_{\varepsilon}\right)$ for $k=0,1, \ldots, n$. Then, by applying $n+1$ times Theorem 3.2

$$
T_{k}^{\prime}(s)=-\left.\int_{0}^{T_{0}} U_{0}\left(\lambda_{k}\right)\right|_{(x, y)=\varphi(t ; s)} d t \text { for } k=1,2, \ldots, n+1,
$$

and consequently, from (a) in Lemma 3.14 ,

$$
d_{k}+3 c_{k}=a_{k}+3 c_{k}=b_{k}-c_{k}=0 \text { for } k=1,2, \ldots, n+1 .
$$

(This follows from the fact that each $T_{k}^{\prime}$ is a linear combination of three functions forming an ECT-system with the coefficients vanishing simultaneously only in case that the above relations hold.) Therefore it turns out that we can write

$$
j^{n+1}\left(X_{\varepsilon}\right)=\left(-y-3 \kappa(\varepsilon) x^{2} y+\kappa(\varepsilon) y^{3}\right) \partial_{x}+\left(x+\kappa(\varepsilon) x^{3}-3 \kappa(\varepsilon) x y^{2}\right) \partial_{y}
$$

with $\kappa(\varepsilon):=1+\sum_{k=1}^{n+1} c_{i} \varepsilon^{k}$. Now, if we define $\Psi_{\varepsilon}(x, y)=(\kappa(\varepsilon) x, \kappa(\varepsilon) y)$, then one can easily verify that it holds $\left(\Psi_{\varepsilon}\right)^{*}\left(j^{n+1}\left(X_{\varepsilon}\right)\right)=X_{0}$. Accordingly $\Phi_{\varepsilon}^{n+1}:=\Phi \circ \Psi_{\varepsilon}$ provides a linearization of the $(n+1)$-jet of $X_{\varepsilon}$. Thus the claim is true.

It is clear that the result under consideration only makes sense in case that the perturbation is not isochronous, so there exists some $\ell \geqslant 0$ such that $T_{0}^{\prime} \equiv T_{1}^{\prime} \equiv \ldots \equiv T_{\ell}^{\prime} \equiv 0$ and $T_{\ell+1}^{\prime} \not \equiv 0$. Then, on account of the claim, there exists an analytic family of linearizations $\left\{\Phi_{\varepsilon}^{\ell}\right\}$ of $j^{\ell}\left(X_{\varepsilon}\right)$ and so, by applying Theorem 3.2 .

$$
T_{\ell+1}^{\prime}(s)=-\left.\int_{0}^{T_{0}} U_{0}\left(\lambda_{\ell+1}\right)\right|_{(x, y)=\varphi(t ; s)} d t \text { for all } s \in \mathcal{I},
$$

Hence, from Lemma 3.14, $T_{\ell+1}^{\prime}(s)=\alpha I_{0}(s)+\beta I_{1}(s)+\gamma I_{2}(s)$ where $\left(I_{0}, I_{1}, I_{2}\right)$ is an ECT-system on $\mathcal{I}$ and $(\alpha, \beta, \gamma)=\phi\left(a_{\ell+1}, b_{\ell+1}, c_{\ell+1}, d_{\ell+1}\right)$ for some surjective linear mapping. Consequently, since $T_{\ell+1}^{\prime}$ is not identically zero, it can have at most two zeros on $\mathcal{I}$. Moreover, since $\phi$ is exhaustive, one can choose $a_{\ell+1}, b_{\ell+1}, c_{\ell+1}$ and $d_{\ell+1}$ such that $T_{\ell+1}^{\prime}(s)=0$ has exactly 0,1 or 2 roots for $s \in \mathcal{I}$ (recall Remark 3.7). This completes the proof of the result.

\section{Proof of Theorem B}

Recall that we consider the unfolding $X_{\varepsilon}=X_{0}+Y_{\varepsilon}$, where $X_{0}$ is the vector field associated to each one of the isochrones in (4) and $Y_{\varepsilon}$ is the perturbation in (5). Note first that, by means of the transformation $(x, y, t) \longmapsto(\eta x, \eta y, \eta t)$ with $\eta=1+b(\varepsilon)$, there is no loss of generality in assuming $b \equiv 0$, i.e., that the unfolding is given by

$$
\left\{\begin{array}{l}
\dot{x}=-y+x y \\
\dot{y}=x+\left(d_{0}+d(\varepsilon)\right) x^{2}+\left(f_{0}+f(\varepsilon)\right) y^{2},
\end{array}\right.
$$

where $d$ and $f$ are analytic functions vanishing at $\varepsilon=0$, and the value of $\left(d_{0}, f_{0}\right)$ is $\left(-\frac{1}{2}, \frac{1}{2}\right),(0,1),\left(1, \frac{1}{4}\right)$ and $\left(-\frac{1}{2}, 2\right)$ in case that we perturb the isochronous center $\left(S_{1}\right),\left(S_{2}\right),\left(S_{3}\right)$ and $\left(S_{4}\right)$, respectively. 
In order to show Theorem $\mathrm{B}$ we must first take, for each isochronous center, a commutator $U_{0}$ of $X_{0}$ defined in the whole period annulus. For instance we can use the ones in [5, 21] but, as it will be clear in a moment, we do not need their concrete expression. Then, as we did in the previous section for the Pleshkan's isochrones, we decompose the perturbation as $X_{\varepsilon}-X_{0}=\lambda X_{0}+\mu U_{0}$. The next result is the counterpart of Lemma 3.14 and it follows after translating the results in [10] to the language that we use here.

Lemma 4.1 (Gasull-Yu). Setting $\lambda=\sum_{i=1}^{\infty} \lambda_{i} \varepsilon^{i}$, define

$$
R(s)=\left.\int_{0}^{T_{0}} U_{0}\left(\lambda_{k}\right)\right|_{(x, y)=\varphi(t ; s)} d t
$$

where $\varphi(t ; s)$ is the solution of $X_{0}$ such that $\varphi(0 ; s)=\xi(s)$ and $\xi: \mathcal{I} \longrightarrow \mathbb{R}^{2}$ is a fixed solution of $U_{0}$. Then

$$
R(s)=d_{k} I_{0}(s)+f_{k} I_{1}(s) \text { for all } s \in \mathcal{I},
$$

where:

(a) $\left(I_{0}, I_{1}\right)$ is an ECT-system on $\mathcal{I}$ in case that $\left(d_{0}, f_{0}\right) \in\left\{(0,1),\left(0, \frac{1}{4}\right),\left(-\frac{1}{2}, 2\right)\right\}$.

(b) $I_{0}(s)=I_{1}(s) \neq 0$ for all $s \in \mathcal{I}$ in case that $\left(d_{0}, f_{0}\right)=\left(-\frac{1}{2}, \frac{1}{2}\right)$.

This result follows from the proof of Theorem 3 in [10, but it is worth making some comments. Recall that to parameterize the periodic orbits we take a commutator $U_{0}$ of $X_{0}$, i.e., such that $\left[X_{0}, U_{0}\right]=0$, analytic in $\mathcal{P} \cup\{(0,0)\}$. This provides a parameterization of the period function of $X_{\varepsilon}$, say $T(s ; \varepsilon)=2 \pi+T_{1}(s) \varepsilon+\mathrm{o}(\varepsilon)$, for which

$$
T_{1}^{\prime}(s)=\left.\int_{0}^{T_{0}} U_{0}\left(\lambda_{1}\right)\right|_{(x, y)=\varphi(t ; s)} d t .
$$

(This expression is well known but it can be viewed as the case $k=0$ of Theorem 3.2.) Instead of a commutator, the authors in [10] use a vector field $\widehat{U}_{0}$ such that $\left[X_{0}, \widehat{U}_{0}\right]=\beta \widehat{U}_{0}$ for some function $\beta$. This yields of course to another parameterization of the period function of $X_{\varepsilon}$, say $\widehat{T}(s ; \varepsilon)=2 \pi+\widehat{T}_{1}(s) \varepsilon+\mathrm{o}(\varepsilon)$. The expression of $\widehat{T}_{1}^{\prime}(s)$ is slightly different from the previous one, but we still can take advantage of their result. Indeed, it is clear that there exists a diffeomorphism $\zeta$ verifying $T(s ; \varepsilon)=\widehat{T}(\zeta(s) ; \varepsilon)$, so that $T_{1}^{\prime}(s)=\zeta^{\prime}(s) \widehat{T}_{1}^{\prime}(\zeta(s))$, see Remark 2.2. The proof of Theorem 3 in [10] shows that $\widehat{T}_{1}^{\prime}(s)=d_{1} \hat{I}_{0}(s)+f_{1} \hat{I}_{1}(s)$, where $\hat{I}_{0}$ and $\hat{I}_{1}$ are functions verifying, for each case, statements $(a)$ and $(b)$ in Lemma 4.1. This is the key point because then

$$
\left.\int_{0}^{T_{0}} U_{0}\left(\lambda_{1}\right)\right|_{(x, y)=\varphi(t ; s)} d t=d_{1} I_{0}(s)+f_{1} I_{1}(s), \text { with } I_{i}(s):=\zeta^{\prime}(s) \hat{I}_{i}(\zeta(s)),
$$

and the result follows due to $\zeta^{\prime}(s) \neq 0$ for all $s$. (To be more precise, this proves the case $k=1$, however the subindex does not play any role at all because $\lambda_{k}(x, y)=F\left(d_{k}, f_{k}, x, y\right)$ with $F$ not depending on $k$.)

Let us note that analogous computations to the ones carried out in the previous section and the application of the criterion given in 12 also yield to the proof of Lemma 4.1. For the sake of shortness we prefer to take advantage of the results in [10] instead.

Proof of Theorem B Let us fix any $\left(d_{0}, f_{0}\right) \in\left\{(0,1),\left(0, \frac{1}{4}\right),\left(-\frac{1}{2}, 2\right)\right\}$ and consider the unfolding $X_{\varepsilon}$ in (9) of the isochronous center $X_{0}$. Let us also take the corresponding commutator $U_{0}$ of $X_{0}$ and, as usual, denote the period function of $X_{\varepsilon}$ using a solution of $U_{0}$ as transversal section by $T(s ; \varepsilon)$. Finally, let $\Phi$ be a linearization of $X_{0}$.

We claim that if $T_{0}^{\prime} \equiv T_{1}^{\prime} \equiv \ldots \equiv T_{k}^{\prime} \equiv 0$, then $j^{k}\left(X_{\varepsilon}\right)=X_{0}$. The proof follows by induction on $k$. The case $k=0$ is trivial because $j^{0}\left(X_{\varepsilon}\right)=X_{0}$. Assume that the claim is true for $k=n$ and let us show its 
validity for $k=n+1$. So suppose that $T_{0}^{\prime} \equiv T_{1}^{\prime} \equiv \ldots \equiv T_{n+1}^{\prime} \equiv 0$ and, by the induction hypothesis, that $j^{n}\left(X_{\varepsilon}\right)=X_{0}$. Then $\Phi$ is a linearization of $j^{k}\left(X_{\varepsilon}\right)=X_{0}$ for $k=0,1, \ldots, n$ and, by applying $n+1$ times Theorem 3.2. we have that

$$
T_{k}^{\prime}(s)=-\left.\int_{0}^{T_{0}} U_{0}\left(\lambda_{k}\right)\right|_{(x, y)=\varphi(t ; s)} d t \text { for } k=1,2, \ldots, n+1 .
$$

Hence, thanks to $(a)$ in Lemma 4.1. $T_{1}^{\prime} \equiv T_{2}^{\prime} \equiv \ldots \equiv T_{n+1}^{\prime} \equiv 0$ implies $d_{k}=f_{k}=0$ for $k=1,2, \ldots, n+1$, so that $j^{n+1}\left(X_{\varepsilon}\right)=X_{0}$. This proves the claim.

It is clear that the result under consideration only makes sense in case that the perturbation is not isochronous, so there exists some $\ell \geqslant 0$ such that $T_{0}^{\prime} \equiv T_{1}^{\prime} \equiv \ldots \equiv T_{\ell}^{\prime} \equiv 0$ and $T_{\ell+1}^{\prime} \not \equiv 0$. Then, on account of the claim, $\Phi$ is a linearization of $j^{\ell}\left(X_{\varepsilon}\right)=X_{0}$ and, by applying Theorem 3.2 .

$$
T_{\ell+1}^{\prime}(s)=-\left.\int_{0}^{T_{0}} U_{0}\left(\lambda_{\ell+1}\right)\right|_{(x, y)=\varphi(t ; s)} d t \text { for all } s \in \mathcal{I} .
$$

Thus, from Lemma 3.14, $T_{\ell+1}^{\prime}(s)=d_{\ell+1} I_{0}(s)+f_{\ell+1} I_{1}(s)$ where $\left(I_{0}, I_{1}\right)$ is an ECT-system on $\mathcal{I}$. Consequently, since $T_{\ell+1}^{\prime}$ is not identically zero, it can have at most one zero for $s \in \mathcal{I}$. Moreover, on account of Remark 3.7 one can choose $d_{\ell+1}$ and $f_{\ell+1}$ such that $T_{\ell+1}^{\prime}(s)=0$ has exactly 0 or 1 root for $s \in \mathcal{I}$. This completes the proof of the result.

We conclude the paper with some final remarks about how Theorem B and Figure 1 fit together. This figure gathers some of the results and conjectures that appear in [19] on the bifurcation diagram of the period function of the so-called dehomogenized Loud's systems

$$
\left\{\begin{array}{l}
\dot{x}=-y+x y \\
\dot{y}=x+D x^{2}+F y^{2}
\end{array}\right.
$$

It may seem surprising that, thanks to Theorem 3.2, it is possible to obtain a result for arbitrary perturbations by taking advantage of a result, namely the one in [10, that holds only for perturbations that are linear in $\varepsilon$. Roughly speaking, this shows that the linear perturbations already give all the critical periods that you can get with an arbitrary perturbation. Certainly this is not a general property but it explains why we could not obtain the result for the perturbation of the isochronous center $\left(d_{0}, f_{0}\right)=\left(-\frac{1}{2}, \frac{1}{2}\right)$, not even for the linear ones. Indeed, a linear perturbation corresponds in the parameter plane to move slightly travelling on a straight line that passes through the isochronous center. In addition if you can get $k \geqslant 0$ critical periods moving in one direction, then you also get $k$ in the opposite one (changing the signum of $\varepsilon$ ). Clearly, see Figure 1, this is what it happens with the isochronous centers $\left(d_{0}, f_{0}\right) \in\left\{(0,1),\left(-\frac{1}{2}, 2\right),\left(0, \frac{1}{4}\right)\right\}$ but not with $\left(d_{0}, f_{0}\right)=\left(-\frac{1}{2}, \frac{1}{2}\right)$. Let us note moreover that if the conjectural diagram in Figure 1 is true, then the bifurcation of $\left(d_{0}, f_{0}\right)=\left(0, \frac{1}{4}\right)$ giving rise to a critical period arising from $\mathcal{P}$ also causes the emergence of another one from $\partial \mathcal{P}$ at the same time. The bifurcation of the latter critical period would be the counterpart, for the period function, of the bifurcation of an alien limit cycle as defined in 2]. (In that paper, the authors present a way to study the perturbations from a Hamiltonian 2-saddle cycle which can produce limit cycles that cannot be detected using zeroes of the Abelian integral, even when it is generic.) On account of Theorem B this, let us say, alien critical period does not come from a zero of the Abelian integral related with the derivative of the period function.

On the other hand, Chicone and Jacobs prove in 3 that there are perturbations of each one of the four isochrones giving rise to exactly one critical period bifurcating from the inner boundary of $\mathcal{P}$. Theorem $B$ shows that there are perturbations of the isochronous centers $\left(d_{0}, f_{0}\right) \in\left\{(0,1),\left(-\frac{1}{2}, 2\right),\left(0, \frac{1}{4}\right)\right\}$ giving rise to exactly one critical period bifurcating from $\mathcal{P}$. These two facts do not contradict the conjecture in Figure 1 near $\left(d_{0}, f_{0}\right)=(0,1)$ and $\left(d_{0}, f_{0}\right)=\left(-\frac{1}{2}, 2\right)$, it simply shows that both perturbations are different. 


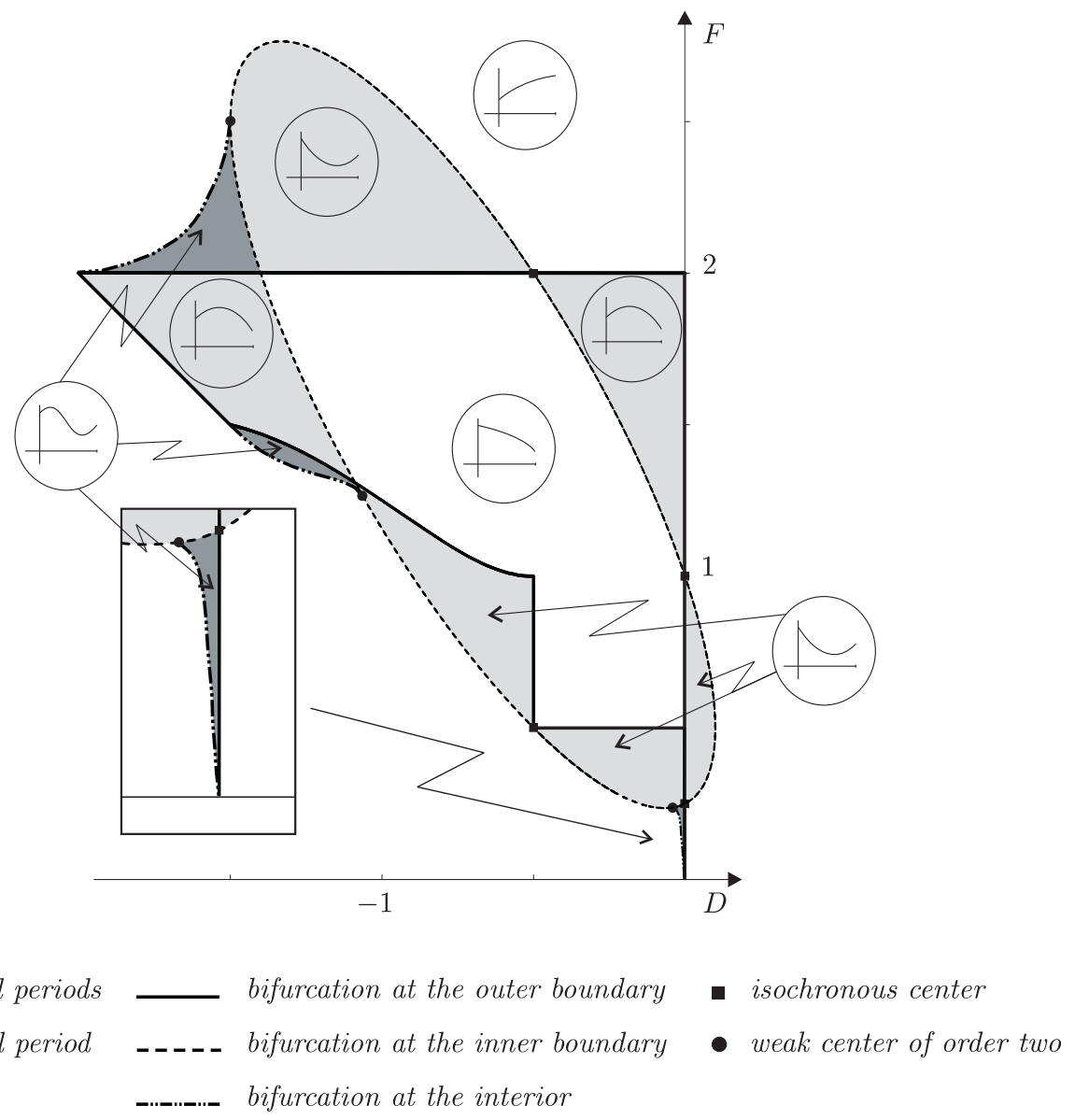

Figure 1: Conjectural bifurcation diagram of the period function of the dehomogenized Loud's systems.

\section{References}

[1] M. Abramowitz and I.A. Stegun, "Handbook of mathematical functions with formulas, graphs, and mathematical tables" Reprint of the 1972 edition. Dover Publications, Inc., New York, 1992.

[2] M. Caubergh, F. Dumortier and R. Roussarie, Alien limit cycles near a Hamiltonian 2-saddle cycle, C. R. Acad. Sci. Paris, Ser. I 340 (2005) 587-592.

[3] C. Chicone and M. Jacobs, Bifurcation of critical periods for plane vector fields, Trans. Amer. Math. Soc. 312 (1989) 433-486.

[4] A. Cima, A. Gasull and P.R. da Silva, On the number of critical periods for planar polynomial systems, Nonlinear Anal. 69 (2008) 1889-1903.

[5] J. Chavarriga and M. Sabatini, A survey of isochronous centers, Qual. Theory Dyn. Syst. 1 (1999) $1-70$.

[6] H. Dulac, Détermination et intégration d'une certaine classe d'équations différentielles ayant pour point singulier un centre, Bull. Soc. Math. France Sér. (2) 32 (1908), 230-252. 
[7] J.-P. Françoise, The successive derivatives of the period function of a plane vector field, J. Differential Equations 146 (1998) 320-335.

[8] E. Freire, A. Gasull and A. Guillamon, Period function for perturbed isochronous centres, Qual. Theory Dyn. Syst. 3 (2002) 275-284.

[9] E. Freire, A. Gasull and A. Guillamon, First derivative of the period function with applications, J. Differential Equations 204 (2004) 139-162.

[10] A. Gasull and Jiang Yu, On the critical periods of perturbed isochronous centers, J. Differential Equations 244 (2008) 696-715.

[11] A. Gasull and Yulin Zhao, Bifurcation of critical periods from the rigid quadratic isochronous vector field, Bull. Sci. Math. 132 (2008) 292-312

[12] M. Grau, F. Mañosas and J. Villadelprat, A Chebyshev criterion for Abelian integrals, preprint (2008) arXiv:0805.1140v2 [math.DS].

[13] S. Karlin and W. Studden, "Tchebycheff systems: with applications in analysis and statistics", Interscience Publishers, 1966.

[14] I. Kolár̆, P. Michor and J. Slovák, "Natural operations in differential geometry", Springer-Verlag, Berlin, 1993.

[15] W.S. Loud, Behaviour of the period of solutions of certain plane autonomous systems near centers, Contrib. Differential Equations 3 (1964) 21-36.

[16] K.E. Malkin, Criteria for center for a differential system, Volzhskii. Matem. Sbornik 2 (1964) 87-91.

[17] P. Mardešić, "Chebyshev systems and the versal unfolding of the cusp of order $n$ ", Travaux en cours, vol. 57, Hermann, Paris, 1998.

[18] P. Mardesic, D. Marín and J. Villadelprat, On time function of the Dulac map for families of meromorphic vector fields, Nonlinearity 16 (2003) 855-881.

[19] P. Mardešić, D. Marín and J. Villadelprat, The period function of reversible quadratic centers, J. Differential Equations 224 (2006) 120-171.

[20] D. Marín and J. Villadelprat, On the return time function around monodromic polycycles, J. Differential Equations 228 (2006) 226-258.

[21] P. Mardešić, L. Moser-Jauslin and C. Rousseau, Darboux linearization and isochronous centers with a rational first integral, J. Differential Equations 134 (1997) 216-268.

[22] I. Pleshkan, A new method of investigating the isochronicity of a system of two differential equations, Differential Equations 5 (1969) 796-802.

[23] C. Rousseau and B. Toni, Local bifurcation of critical periods in vector fields with homogeneous nonlinearities of the third degree, Canad. Math. Bull. 36 (1993) 473-484.

[24] M. Sabatini, Characterizing isochronous centres by Lie brackets, Differential Equations Dynam. Systems 5 (1997) 91-99.

[25] M. Villarini, Regularity properties of the period function near a centre of a planar vector field, Nonlinear Analysis T.M.A. 19 (1992) 787-803. 